\title{
Preparation of Stainless Steel Surfaces for Scanning Probe Microscopy
}

\author{
Alexander D. Warren, ${ }^{1 *}$ Ana I. Martinez-Ubeda, ${ }^{1}$ Oliver \\ Tom B. Scott ${ }^{1}$ \\ ${ }^{1}$ Interface Analysis Centre, HH Wills Laboratory, University of Bristol, Bristol, BS8 1LT, UK \\ ${ }^{2}$ Engineering Maths, Merchant Venture School of Engineering, University of Bristol, Bristol \\ *xander.warren@bristol.ac.uk
}

D. Payton, ${ }^{1,2}$ Loren Picco, ${ }^{1}$ and

\begin{abstract}
A surface preparation route is presented that is designed to give high-quality finishes to austenitic stainless steels for analysis with advanced scanning probe microscopy techniques. The method details a series of polishing and cleaning steps suitable for novices and experts alike. The steps taken are justified throughout and illustrated with examples of potential defects.
\end{abstract}

\section{Introduction}

The properties of metals and alloys depend on their microstructure, including grain size, number of phases, amounts of phases, and phase distributions within the sample at the micrometer scale. Microstructure is elucidated through metallographic examination. Typical preparation steps for specimens of this type include mounting in a thermosetting resin, polishing to a mirror-like finish, and in some cases etching with a weak acid (chosen to selectively dissolve grain boundaries or specific phases). Conventional techniques used for subsequent examination include light optical microscopy (LOM), scanning electron microscopy (SEM), and electron backscatter diffraction (EBSD). The latest developments in metallographic analysis are focused at the nanometer scale and require precise sample preparation. Scanning probe microscopy (SPM) techniques such as atomic force microscopy (AFM) are well-suited for the analysis of metallographic features at sub-micrometer length scales including the following: precise determination of carbide morphology, topographic analysis of creep cavities and corrosion, and determination of phase distributions using stiffness or magnetic data [1]. The suite of SPM techniques are non-destructive, allowing the same area of interest to be examined later by other techniques. This saves considerable time compared with destructive metallographic techniques and enables direct comparisons of the resulting data.

In the experience of the authors, there is a degree of confusion regarding the most effective way to prepare stainless steel specimens to achieve a final finish suitable for SPM analysis. Although sample preparation notes for techniques such as EBSD imaging have been published [2], they often fail to specify cleaning methods to remove the fine layers of organic material and stray particles (for example, colloidal silicon or diamond), which sequential polishing typically generates. Such preparation procedures are sufficient for sample inspection with techniques where the information is coming mainly from a volume beneath the actual surface (for example, SEM, BSE, EBSD, etc.) because nano-scale surface contamination does not significantly distort the signal, and the contamination is often not observed. However AFM, like many SPM techniques, requires near-pristine specimen surfaces to maximize results, and conventional metallographic preparation may not be suitable. If the specimen or SPM probe tip becomes contaminated with an organic residue, such as that left by some polishing lubricants, the recorded data could be distorted [3-5].

In this article we set out a methodology that has been found to be effective in the preparation of AISI Type 300 series austenitic and SAF 2205 (duplex) stainless steels. The preparation route described has been shown to give a reproducible high-quality surface polish, with the cleaning stages being effective at removing residues and particulates. The principal differences compared with other sample preparation procedures [2] are the time spent on each grinding/polishing step, the number of fresh $\mathrm{SiC}$ papers used, and the rigor of the post-polishing sample cleaning. It is important not to skip any step because each is required to remove mechanical damage induced by the previous step. Although the technique minimizes preferential polishing on softer phases, this will still occur to some degree and lead to a higher variation in surface topography, particularly in fine-grained or heavily aged precipitation-hardening materials. The latter is beyond the scope of this paper.

\section{Materials and Methods}

Cut the sample to size using an automated diamond disc cut-off saw (for example, a Struers Accutom - Struers, Rødovre, Denmark) with the sample fed at $0.05 \mathrm{~mm} / \mathrm{min}$ to minimize damage to the cut surfaces. The sample is then set in resin (cold set, non-adhesive resins are preferred (for example, Struers Clarocit)) prior to polishing. The use of a programmable automatic polisher (for example, Struers Tegrapol-15 polisher) with the sample holder rotating in the same direction as the
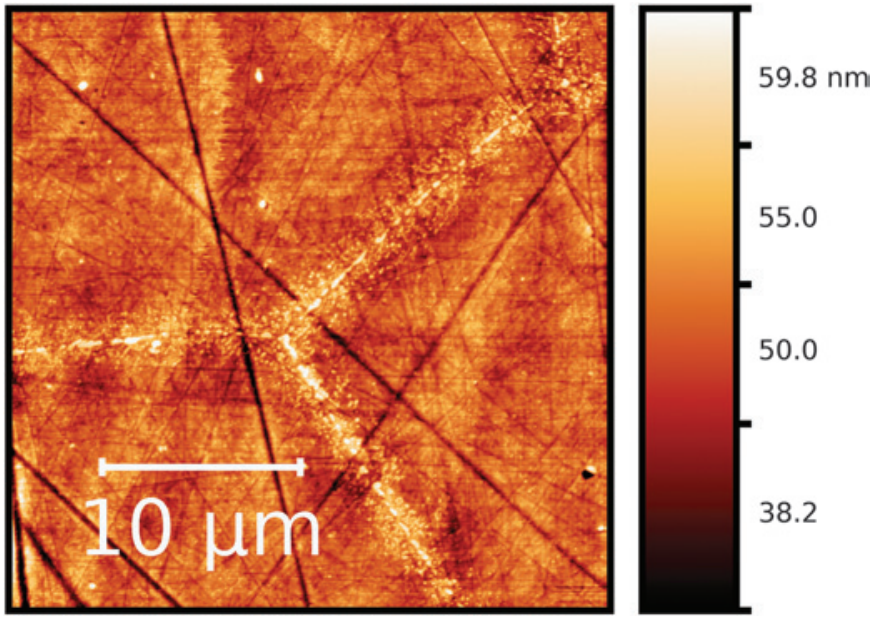

Figure 1: Topographic AFM map showing a specimen with large scratches caused by a poor coarse polish. Later stages of polishing were more thorough, revealing the grain boundary precipitation in the center. 
Visit the meeting website often for details on

Symposia, Exhibits, Biological and Physical Sciences Tutorials,

$$
\text { In-Week Intensive Workshops, Posters, }
$$

Sunday Short Courses and Pre-Meeting Congresses
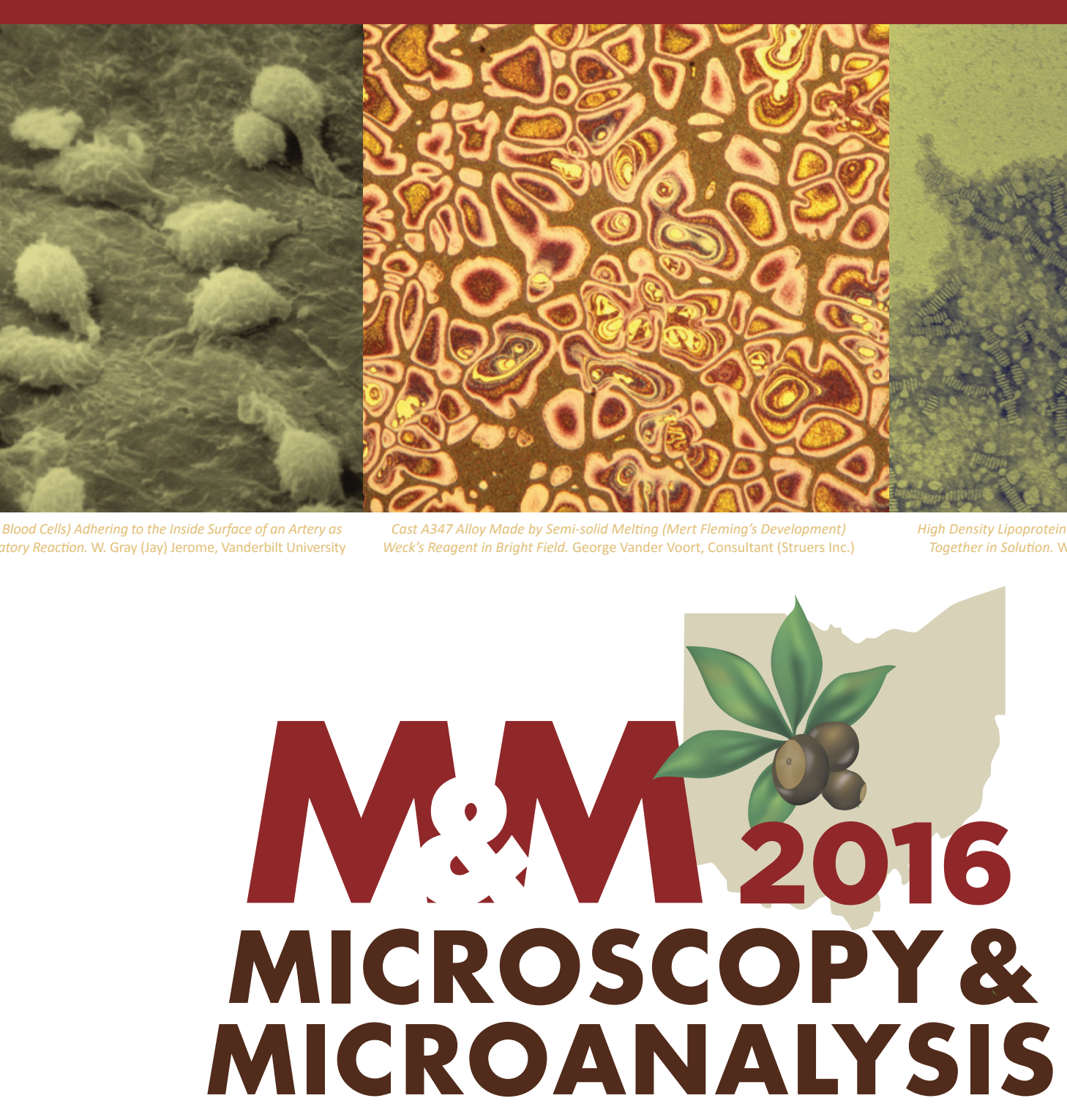

July 24-28, 2016 - Columbus, Ohio

\section{http://microscopy.org/MandM/2016}

Program Information | Information for Students | Meeting Awards | Exhibitor List \& Floor Plan Registration (opens February 2016) | Columbus Hotel Information (reservations available February 2016) 


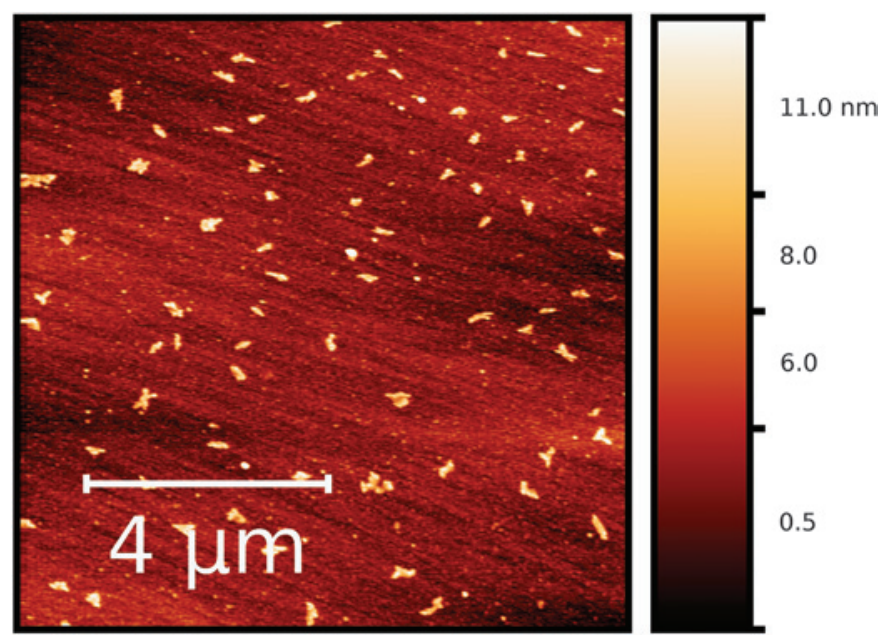

Figure 2: Topographic AFM map showing a steel surface with a good coarse polish, but the overall result was marred by insufficient diamond polishing (which should have removed the $\sim 2 \mathrm{~nm}$ deep scratches) and salt crystals formed during the drying of tap water.

polishing pad is recommended to maintain reproducibility in applied pressure compared to manual polishing.

Polishing steps. The following steps are recommended for AISI 300 series and SAF 2205 stainless steels:

1. $60 \mathrm{~s}$ at $300 \mathrm{rpm}$, and $30 \mathrm{~N}$, on 320-Grit SiC paper with water lubricant. This step is necessary to remove excess resin from the face of the sample.

2. $60 \mathrm{~s}$ at $300 \mathrm{rpm}$, and $30 \mathrm{~N}$, on $800-\mathrm{Grit} \mathrm{SiC}$ paper with water lubricant.

3. 3 minutes at $300 \mathrm{rpm}$, and $30 \mathrm{~N}$, on $1200-\mathrm{Grit} \mathrm{SiC}$ paper with water lubricant.

4. 5 minutes at $300 \mathrm{rpm}$ and $30 \mathrm{~N}$ on $2400-\mathrm{Grit} \mathrm{SiC}$ paper with water lubricant.

5. 10 minutes at $150 \mathrm{rpm}$ and $30 \mathrm{~N}$ on $4000-\mathrm{Grit} \mathrm{SiC}$ paper with water lubricant.

6. 5 minutes at $150 \mathrm{rpm}$ and $15 \mathrm{~N}$ on $4000-\mathrm{Grit} \mathrm{SiC}$ paper with water lubricant.

7. 10 minutes polishing by hand, using $1 \mu \mathrm{m}$ diamond paste on a nylon cloth and aerosol lubricant (for example, Hyprez fluids, Engis Ltd., Maidstone, UK). The direction of polishing should be varied throughout the process.

8. 10 minutes polishing by hand, using $1 / 4 \mu \mathrm{m}$ diamond paste on a nylon cloth and aerosol lubricant (for example, Hyprez fluids, Engis Ltd., Maidstone, UK). The direction of polishing should be varied throughout the process.

9. 24 hours on a Vibro-polisher (Buehler Vibromet 2 Buehler/Illinois Tool Works Inc., Lake Bluff, Illinois), at a rotational speed of $3 \mathrm{rpm}$ with an oxide polishing suspension (OPS).

Each time the grinding pad is changed, both the sample and sample holder should be removed from the grinder and given a thorough wash with dionized (DI) water and detergent, followed by rinsing with methanol and blow-drying using $\mathrm{N}_{2}$. If samples are particularly delicate, it is suggested that steps I and II be skipped and step III repeated until the polishing breaks through the resin.

Between each of the last 3 polishing steps, the specimen should be washed twice with DI water and detergent using a lint-free cloth before being rinsed with isopropanol and

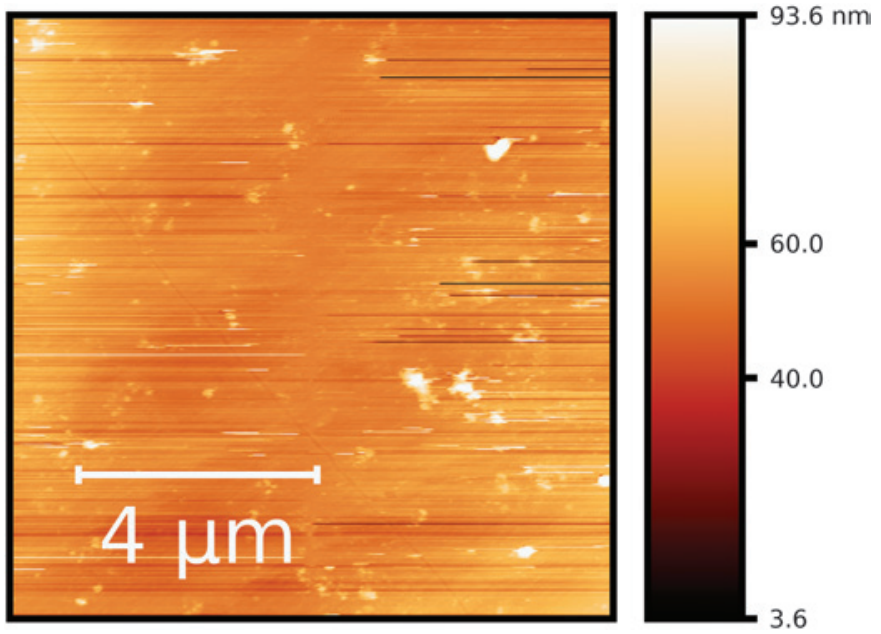

Figure 3: Topographic AFM map showing a well-polished sample. The streaking is generated by the movement of loose particles/resin-debris, resulting from ineffective specimen cleaning following the removal of the resin mount.

blow-dried using dry $\mathrm{N}_{2}$. These washes are important to avoid cross-contamination of the polishing pastes. Figure 1 shows a specimen with a poor initial polish (leaving large scratches), but more effective polishing at later stages revealed grain boundary precipitation.

Cleaning steps. After the final step in the procedure, it is necessary to rinse the specimen thoroughly with de-ionised (DI) water and detergent. Many SPM applications require a small specimen size, and as such it may be necessary to remove
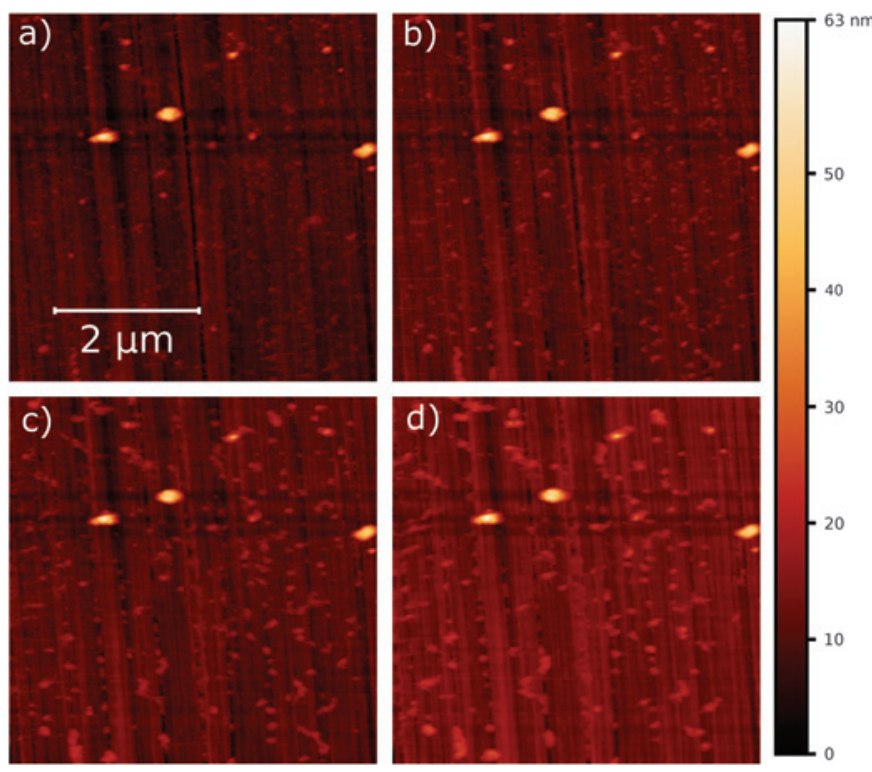

Figure 4: Shows sequential frames from a high-speed AFM of a steel sample that has been incorrectly prepared for AFM imaging. Scratch marks due to partial polishing running vertically are clearly visible, as is surface contamination. Organic material, present as a film in (a) is collected up by the tip as it passes over the surface and is redistributed, infilling sample topography and presenting itself as positive topological features. Frames (a)-(d) are taken at $0 \mathrm{~s}, 0.5 \mathrm{~s}, 1 \mathrm{~s}$, and $5 \mathrm{~s}$ after moving to a new location on the sample at 2 frames per second. Organic material can be hard to spot in a single image, but when frames are combined to form a video or time lapse, the motion of the organic material due to contact with the probe is obvious. The organic material also adheres itself to the tip thus reducing the resolution of the image. 


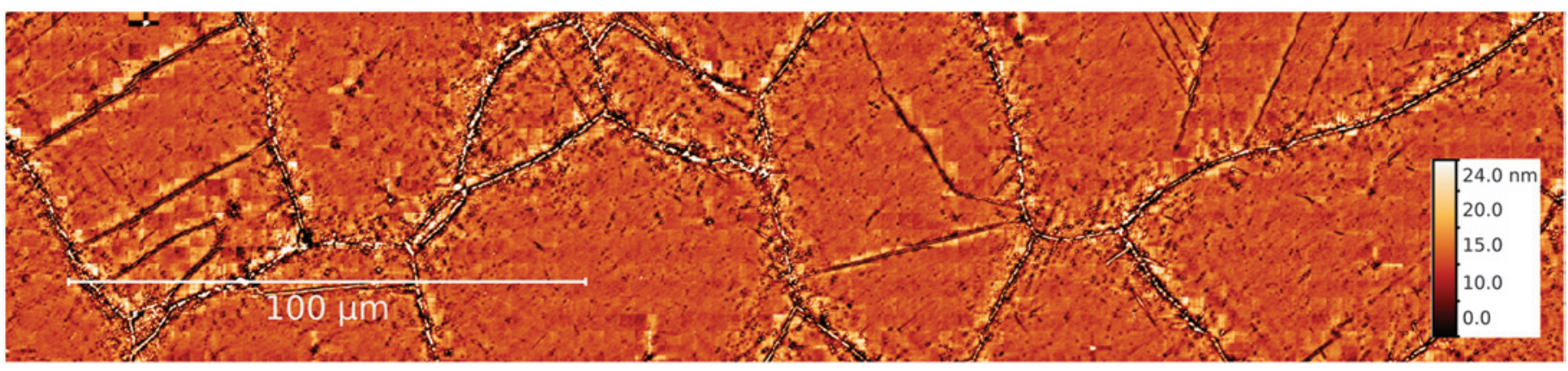

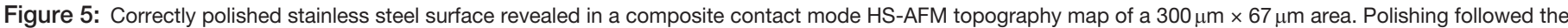

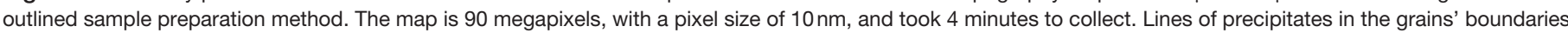
are shown with a height of about $10 \mathrm{~nm}$ above the average plane of the surface.

the sample from the resin puck. This should be done now, as no further polishing is required. Depending on resin type, it may be possible to dissolve the resin, or it may need to be cut away. For thin samples mounted in non-adhesive resin, typically it is only necessary to carefully cut around the edge of the sample using a scalpel or razor blade. For thicker samples, it may be necessary to reduce the size of the resin mount using a hack-saw and vice, before removing remaining resin from the sample with a scalpel or fine-nose wire cutters, taking care not to touch the polished face. Where possible, avoid getting dust/ resin residue on the specimen face, and always use gloves and tweezers for specimen manipulation to reduce the potential for contamination. Rinse the specimen again with DI water followed by sonicating with acetone to remove any trace resin particles, then blow dry with $\mathrm{N}_{2}$. Figure 2 shows an effective polish, but salt particles have formed during drying because standard tap water was used and no blow-drying was employed. Figure 3 shows the streaking caused by movement of loose debris, such as resin dust or residual colloidal silicon particles, across a well-polished specimen surface.

Using a clean beaker, sonicate the specimen in acetone for 2 minutes. Thoroughly clean the beaker and specimen with sequential washes of DI water and detergent, ethanol, and acetone. Repeat the acetone sonication. As most resins are soluble in acetone, this two-step sonication is necessary to dissolve any resin residues adhering to the specimen. Next, clean the specimen and beaker using the same sequential washing process as before and repeat the sonication process with ethanol. Clean the specimen and beaker using the same sequential washing process as before and repeat the sonication process with isopropanol. The use of organic solvents is important to ensure the thorough removal of any oil-based residues left from diamond polishing (Figure 4). Clean the specimen and the beaker and repeat the sonication with DI water. Wash the sample thoroughly with isopropanol and DI water, followed by blow-drying with $\mathrm{N}_{2}$ to prevent the formation of drying marks.

Storing and mounting for AFM. Store the specimen in a dust-free environment to ensure no contamination prior to analysis. Sealable plastic bags should not be used, as it is possible for some of the plastic to transfer onto the sample and affect the quality of the surface. When mounting the sample onto the SPM, do not use cyanocrylate-based glues (superglues) because the vapor given off can settle on the sample. Figure 5 shows a composite contact mode high-speed AFM [6] image of a large area of steel that has been correctly prepared using the method described, revealing no surface debris or scratches but exhibiting significant grain boundary precipitation inherent in the sample. A contact mode high-speed AFM [7] was used to assess areas of samples far larger than can be imaged using a traditional AFM. This is achieved by stitching together the video rate images collected using the high-speed AFM as the microscope moves across the surface. The resulting image has the same lateral resolution as a conventional AFM but is capable of imaging over millimeter-sized areas.

\section{Conclusion}

SPM images are especially sensitive to artifacts arising from surface contamination. The method described in this article has been used to prepare a range of steels and has been shown to work consistently over large sample areas. High-speed AFM (HS-AFM) data show that with correct sample preparation, steel samples can be prepared that are free from scratches, debris, and organic and salt contamination over areas millimeters in size whilst leaving material features such as grain boundaries visible.

\section{Acknowledgements}

Author ADW wishes to acknowledge the Engineering and Physical Sciences Research Council PROMINENT consortium [EP/I003282/1] for funding; AMU was supported by EDF Energy; and authors OP and LP were both funded by the Royal Academy of Engineering. The authors would also like to thank EDF Energy and Outukompu steel for provision of the sample materials and the University of Bristol's NSQI Centre for hosting the high-speed AFM.

\section{References}

[1] AD Warren et al., Ultramicroscopy 148, (2015) 1-9.

[2] MM Nowell et al., Microsc Microanal 11(S02) (2005) 504-05.

[3] U Hubner et al., Appl Phys A-Mater 76 (2003) 913-17.

[4] Y-S Lo et al., Langmuir 15 (1999) 6522-26.

[5] J Vesenka et al., Rev Sci Instrum 65 (1994) 2249.

[6] P Klapetek et al., Nanotechnology 26 (2015) 065501-10.

[7] O Payton et al., Nanotechnology 23 (2012) 205704-10. 